\title{
Toward an online cognitive and emotional battery to predict treatment remission in depression
}

\author{
This article was published in the following Dove Press journal: \\ Neuropsychiatric Disease and Treatment \\ 26 February 2015 \\ Number of times this article has been viewed
}

\section{Evian Gordon' \\ A John Rush² \\ Donna M Palmer ${ }^{3,4}$ \\ Taylor A Braund ${ }^{3}$ \\ William Rekshan'}

'Brain Resource, San Francisco, CA, USA; ${ }^{2}$ Duke-NUS, Singapore; ${ }^{3}$ Brain Resource, Sydney, NSW, Australia; ${ }^{4}$ Brain Dynamics Center, Sydney Medical School - Westmead and Westmead Millennium Institute, The University of Sydney, Sydney, NSW, Australia
Correspondence: Evian Gordon Brain Resource, II 5 Sansome St, Suite 600-B, San Francisco, CA 94104, USA $\mathrm{Tel}+\mathrm{I} 4153997990$

Fax + I 4I5852 5198

Email eviang@brainresource.us
Purpose: To evaluate the performance of a cognitive and emotional test battery in a representative sample of depressed outpatients to inform likelihood of remission over 8 weeks of treatment with each of three common antidepressant medications.

Patients and methods: Outpatients 18-65 years old with nonpsychotic major depressive disorder (17 sites) were randomized to escitalopram, sertraline or venlafaxine-XR (extended release). Participants scored $\geq 12$ on the baseline 16-item Quick Inventory of Depressive Symptomatology - Self-Report and completed 8 weeks of treatment. The baseline test battery measured cognitive and emotional status. Exploratory multivariate logistic regression models predicting remission (16-item Quick Inventory of Depressive Symptomatology - Self-Report score $\leq 5$ at 8 weeks) were developed independently for each medication in subgroups stratified by age, sex, or cognitive and emotional test performance. The model with the highest crossvalidated accuracy determined the participant proportion in each arm for whom remission could be predicted with an accuracy $\geq 10 \%$ above chance. The proportion for whom a prediction could be made with very high certainty (positive predictive value and negative predictive value exceeding $80 \%$ ) was calculated by incrementally increasing test battery thresholds to predict remission/non-remission.

Results: The test battery, individually developed for each medication, improved identification of remitting and non-remitting participants by $\geq 10 \%$ beyond chance for 243 of 467 participants. The overall remission rates were escitalopram: $40.8 \%$, sertraline: $30.3 \%$, and venlafaxine-XR: $31.1 \%$. Within this subset for whom prediction exceeded chance, test battery thresholds established a negative predictive value of $\geq 80 \%$, which identified $40.9 \%$ of participants not remitting on escitalopram, $77.1 \%$ of participants not remitting on sertraline, and $38.7 \%$ of participants not remitting on venlafaxine-XR (all including 20\% false negatives).

Conclusion: The test battery identified about $50 \%$ of each medication group as being $\geq 10 \%$ more or less likely to remit than by chance, and identified about $38 \%$ of individuals who did not remit with $\geq 80 \%$ certainty. Clinicians might choose to avoid this specific medication in these particular patients.

Keywords: depression, treatment selection, cognitive tests, biomarkers, treatment prediction, antidepressant medication

\section{Introduction}

Major depressive disorder (MDD) is a disabling, life shortening, and costly disorder that affects over $15 \%$ of the population. ${ }^{1}$ In terms of burden of disease, depression is the third most disabling medical condition worldwide, and is projected to be the first in developed nations by 2030 . $^{2,3}$

Antidepressant medications are commonly used to treat depression. Remission is the goal of treatment, ${ }^{4}$ yet less than $50 \%$ of patients achieve remission with their initial treatment. $^{5-7}$ Presently, selecting the best treatment for depressed patients rests on a trial 
and error approach, with no clinically useful guidance as to which treatment is preferred for any individual patient. ${ }^{8}$ Overall, patients with more severe depression, ${ }^{9}$ more concurrent general medical conditions, ${ }^{10}$ or more concurrent psychiatric disorders, ${ }^{11,12}$ are more likely to not achieve remission during acute phase treatment. These features can differentiate between groups of patients who do and do not remit, hereby allowing risk stratification. However, these features are not sufficiently specific to allow the identification of individual patients who will or will not remit with enough certainty that clinical decisions can be made..$^{13,14}$

If one could better predict whether a particular treatment is quite likely or quite unlikely to work for a particular patient, substantial savings in time, effort, cost, and patient suffering would follow. ${ }^{15,16}$ While several reports have begun to suggest that pretreatment tests could inform the selection of treatment, ${ }^{17,18}$ none have yet entered clinical care. ${ }^{19-21}$ Cognitive and emotional tests are particularly well suited for use in the selection of treatment as these are scalable and guidance could be provided instantly at the point of care.

This report evaluated the performance of a well established $^{22}$ and validated cognitive and emotional test battery $^{23-25}$ in a representative group of depressed outpatients as part of the International Study to Predict Optimized Treatment in Depression (iSPOT-D) trial ${ }^{26}$ to address several questions. The primary question was: can this test battery identify a meaningful proportion of these patients that exceeds chance prediction (defined as a 50-50 likelihood of remission) by at least $10 \%$ in depressed patients who are treated for 8 weeks with escitalopram, sertraline or venlafaxine-XR (extended release). To further explore the performance of the test battery, our secondary question was whether the test battery could identify a meaningful proportion of patients who, with substantial certainty (at least $80 \%$ ), will either achieve or not achieve remission with 8 weeks of escitalopram, sertraline or venlafaxine-XR? This report serves to put forth preliminary hypotheses that are to be evaluated in an independent sample of depressed patients. ${ }^{26}$

\section{Methods}

\section{Overview}

Details of the iSPOT-D study design and procedures have been reported elsewhere. ${ }^{26}$ In short, in this multiple-phase, multi-site trial, outpatients with nonpsychotic MDD were randomly assigned to one of three antidepressants: escitalopram, sertraline or venlafaxine-XR. Participants in this report were recruited from 17 sites (in five countries) consisting of eight academic sites (four with outpatient psychiatry services, three with outpatient psychology services, and one with combined psychiatry and primary care services) and nine private sites (four psychology/primary care, four psychology, and one psychiatry services).

This report entails a preliminary evaluation of a pretreatment test battery in participants randomized to 8 weeks of treatment with one of these three medications to determine whether the test battery will identify individual depressed patients in each treatment group who will or will not remit in this time period.

\section{Study participants}

The study was approved by Institutional or Ethic Review Boards. Compliance with International Conference on Harmonization and Good Clinical Practice principles, the US Food and Drug Administration Code of Federal Regulations, and country-specific guidelines were implemented. Participants $(\mathrm{N}=1,008)$, all of whom provided written informed consent, were adults (18-65 years old) with a current diagnosis of single-episode or recurrent, nonpsychotic, MDD. Table S1 further details the inclusion and exclusion criteria. The Mini-International Neuropsychiatric Interview was used to establish Diagnostic and Statistical Manual of Mental Disorders (DSM)-IV diagnoses. The full iSPOT study ${ }^{25}$ required a 17 -item Hamilton Rating Scale for Depression $\left(\mathrm{HRSD}_{17}\right)$ score $\geq 16$ at entry. All enrollees were either antidepressant-naïve or had undergone a washout period of at least five half-lives of a previously prescribed antidepressant. All participants were aware of the medication that they were taking.

Because this report aimed to develop an algorithm that could predict remission after 8 full weeks of antidepressant treatment for a meaningful proportion of depressed patients, and because the primary outcome was remission defined by the 16-item Quick Inventory of Depressive Symptoms - SelfRated (QIDS-SR ${ }_{16}$ ) at 8 weeks, this particular report focused exclusively on participants who completed all 8 weeks and who entered the study with a QIDS-SR ${ }_{16}$ score $\geq 12$ (equivalent to an $\mathrm{HRSD}_{17}$ score $\geq 16$ [www.ids-qids.org]) and who had completed at least $50 \%$ of the cognitive and emotional test battery. The focus on completers will introduce bias into the analytic sample. However, the alternative, the use of imputation, suffers from unreliability in declaring remission with certainty for each participant. Since this initial report was designed to generate hypotheses that would later be tested in a replication sample, we chose to focus only on the per protocol patients who completed 8 weeks. In this context, current results do not establish the performance of the 
algorithm in actual practice. From the initial starting sample of 336 patients in each treatment arm, 157, 175, and 135 per protocol patients who completed the study were analyzed for escitalopram, sertraline, and venlafaxine-XR, respectively.

We also performed the same cognitive and emotional test battery on 336 age-, sex- and education-matched healthy controls at baseline and 8 weeks. ${ }^{25}$

\section{Test battery}

The cognitive assessment battery included eight general cognition tasks and one emotional cognition task (Table 1). These nine tests yield 18 measures of cognitive performance. For further details of the cognitive assessment tasks and construct validity see Gordon et al, ${ }^{22}$ Paul et $\mathrm{al}^{23,24}$ and Williams et $\mathrm{al}^{25}$.

\section{Statistical analyses}

We first developed classification models to predict remission for each treatment arm to identify the proportion of depressed outpatients for whom the cognitive and emotional function test battery could provide predictions of remission with performance exceeding chance. Due to the heterogeneity of $\mathrm{MDD},{ }^{27}$ we assumed that one test would not apply to all MDD patients. Therefore, exploratory multivariate logistic regression models to predict remission were developed in the entire sample and separately for subgroups of participants stratified by age, sex, symptom severity, and nine different metrics of cognitive and emotional performance. The median value of each measure (aside from sex) was used to stratify the participants, resulting in two groups for each measure. Age, sex, baseline depression severity (assessed by QIDS-SR ${ }_{16}$ ), three tests from the cognitive and emotion battery (verbal interference reaction time, number of errors on the maze task, and go/no-go reaction time), and six different summary metrics of the cognitive and emotion test battery were used to stratify the sample, resulting in 12 different stratification metrics. The cognitive and emotion tests and summary metrics were used because each was observed to have a kappa $>0.5$ in the iSPOT-D healthy control sample ( $n=336$, with 8 weeks between tests). The summary metrics used to stratify the sample were not used as predictors in the multivariate analysis because the multivariate analysis seeks to, by definition, combine many different tests in an optimal way to predict a dependent variable. Overall, we evaluated 12 different methods of stratifying the participants, resulting in a total of 24 different participant subgroups.

Logistic regression methods were chosen because they are simple, more transparent than black box algorithms, and perform well in many applications of binary predictions. Logistic regression has performed well in real world comparisons of data mining methods using neuropsychological testing. ${ }^{28}$ Due to the exploratory nature of this analysis, the accuracy statistics (accuracy, sensitivity, specificity, positive predictive values [PPV], and negative predictive values [NPV]) were reported for all available data and were cross-validated.

For each analysis, the following steps were followed for model development:

- Step 1: clean and normalize all predictors relative to the iSPOT-D healthy control sample $(\mathrm{N}=336) .{ }^{26}$ Outliers, defined as greater or less than 3 standard deviations away from the mean, were replaced with the largest/smallest non-outlier value. Cognition, age, sex, and symptom severity measures were transformed to $z$-scores relative

Table I Cognitive and emotion tasks

\begin{tabular}{|c|c|c|c|}
\hline Task name & Assesses & Test duration & Measures of task performance \\
\hline \multicolumn{4}{|l|}{ General cognition tasks } \\
\hline Switching of attention & Information processing speed & 3 minutes & Completion time \\
\hline Verbal interference & Cognitive flexibility & 2 minutes & Reaction time - incongruent trials \\
\hline Continuous performance test & Attention & 6 minutes & Reaction time \\
\hline Go/no-go & Response inhibition & 4 minutes & Reaction time \\
\hline Digit span & Working memory & 6 minutes & Total number of trials correct \\
\hline \multirow[t]{2}{*}{ Motor tapping } & Motor coordination & I minute & Number of taps - dominant hand \\
\hline & & & $\begin{array}{l}\text { Number of taps - non-dominant } \\
\text { hand }\end{array}$ \\
\hline \multirow[t]{3}{*}{ Maze } & Executive function & 10 minutes & Completion time \\
\hline & & & Number of errors \\
\hline & & & Number of overrun errors \\
\hline \multicolumn{4}{|l|}{ Emotional cognition tasks } \\
\hline \multirow[t]{2}{*}{ Emotion identification } & Emotion identification accuracy & 4 minutes & Number of correct responses \\
\hline & Speed of emotion identification & & Reaction time for correct responses \\
\hline
\end{tabular}


to the healthy control group. All cognitive performance $z$-scores of reaction time or task completion time were corrected for direction (multiplied by -1 ) so that higher values reflect better (ie, faster) performance.

- Step 2: to avoid co-linearity, identify and remove all highly correlated predictor measures $(r>0.70)$ by removing the predictor with the highest mean absolute correlations with all of the other predictors being evaluated.

- Step 3: to achieve a test with sufficient clinical value while minimizing patient burden, the 3,4 , or 5 predictors with the smallest $P$-values using univariate logistic regression analysis were identified.

- Step 4: perform multivariate logistic regression analysis using the predictor features selected in Step 3, including both main and all possible two-way interaction effects. The model was developed using all available participants who met the entry criteria and criteria for the specific subgroup. A random sample of $80 \%$ of the data was used to develop the regression model during cross-validation.

This was cross-validated by repeating this process 300 times, each time selecting a random $80 \%$ of the sample allocated to train the model and using the remaining $20 \%$ of the data to test the model. The cross-validated regression models were evaluated independently for each treatment arm. All cross-validated accuracy statistics are the average of the performance of the predictions of the test set for the 300 rounds of cross-validation. Of the models that achieved a cross-validated accuracy of 10 percentage points above chance, the final reported models were selected to achieve the maximum cross-validated accuracy. Because cross validation will only provide an assessment of the generalizability of a model for a specific set of data and set of statistical manipulations, a single model must be developed in order to provide a single prediction for individual patients. Therefore, once the regression models and subgroups were identified in the cross validated analyses, new regression models were developed using all available data for the subgroup and treatment arm identified in the cross-validated analyses to allow for both additional calculations regarding performance (see below) and potential application in the real world.

In order to determine the proportion of participants for whom a prediction of remission or non-remission could be made with very high certainty (PPV and NPV exceeding $80 \%$ ), the predicted probability of remission was obtained from the regression models developed using all available data for the subgroup and treatment arm (not cross-validated). These values range from 0 (no probability of remission) to 1 (maximum probability of remission). The participants were subset according to predicted probability of remission, incrementally by 0.01 probability of remission. NPV and PPV were calculated for each subset of participants and the largest number of participants for whom a prediction could be made with an NPV/PPV exceeding $80 \%$ was used to determine the proportion of participants for whom a prediction could be made with high certainty. This process was conducted separately for each treatment arm and for only participants in the specific subgroup identified in the cross-validated analyses.

All analyses were conducted using R 3.0.2. ${ }^{29}$ Highly correlated predictors were identified using the caret package of $\mathrm{R},{ }^{30}$ receiver operating characteristics curves were drawn using the Epi package of $\mathrm{R},{ }^{31}$ and pie charts were generated using the ggplot2 package of $\mathrm{R}^{32}$

\section{Results \\ Overview}

Figure 1 (Consort chart) shows the formation of the analyzable sample. As expected, the sample used for this analysis presented with greater depression severity, longer duration of the depressive disorder (current age minus age at first episode), lower quality of life, and more concurrent general medical conditions compared to excluded participants. (See Tables S2 and S3 for all baseline demographic, severity, and clinical comparisons).

Overall, 157 analyzable participants completed 8 weeks on escitalopram; 175 on sertraline, and 135 on venlafaxine-XR. Remission rates at 8 weeks for these samples were 64/157 (40.8\%) for escitalopram, 53/175 (30.3\%) for sertraline, and $42 / 135$ (31.1\%) for venlafaxine-XR. The proportion of remitters did not significantly vary across treatment arms $\left(\chi^{2}(2, \mathrm{~N}=467)=4.78, P=0.09\right)$.

\section{Question I}

Can this test battery identify a meaningful proportion of these participants that exceeds chance prediction (defined as 50-50 likelihood of remission) by at least $10 \%$ for depressed participants who are treated for 8 weeks with escitalopram, sertraline or venlafaxine-XR?

We found that we could predict remission in each medication arm with a cross-validated estimate of accuracy - as well as sensitivity and specificity - that exceeded chance by 10 percentage points for nearly half of the final sample for each arm. Only when the participants in each arm were subgrouped according to performance on cognitive or emotion tests did the predictions of remission exceed 10 percentage points above chance.

Table 2 shows the percentage of participants in each treatment arm for whom a prediction of remission, both likely 


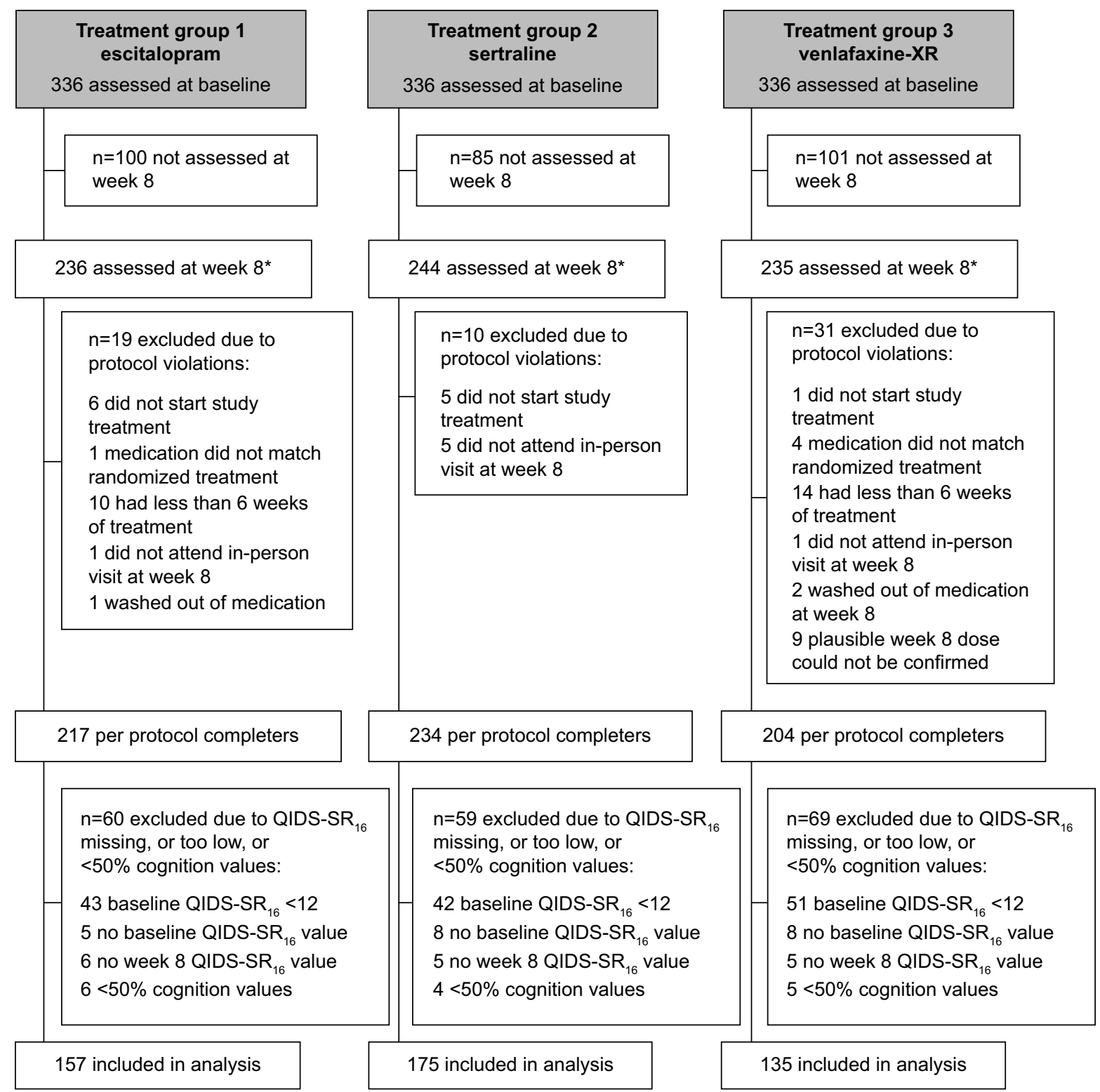

Figure I Consort chart.

Notes: Of the patients who met inclusion criteria for the iSPOT-D study, additional criteria were used for this specific analysis. *Minimum criteria for assessment at week 8 is either completion of the $\mathrm{HRSD}_{17}$ or SOFAS assessments at the in-person visit, or completion of the week 8 QIDS-SR $_{16}$ in the online questionnaire at home.

Abbreviations: QIDS-SR ${ }_{16}$, 16-item Quick Inventory of Depressive Symptomatology Self-Report; iSPOT-D, International Study to Predict Treatment Response in Depression; XR, extended release; HRSD 17 , 17-item Hamilton Rating Scale for Depression.

and unlikely to remit, could be made. The test battery was able to make predictions of remission in $43.3 \%(68 / 157)$ of participants in the escitalopram arm, 63.4\% (111/175) of those in the sertraline arm, and $47.4 \%(64 / 135)$ of those in venlafaxine-XR arm. If we applied all three tests in the test battery to all participants in the three treatment arms, nearly $96 \%$ of participants would receive a prediction of remission/ non-remission on at least one of the three treatments.

Table 3 reports the performance of each classification model for each treatment arm in specific subgroups of

Table 2 Proportion of patients by treatment for whom a test battery recommendation could be made

\begin{tabular}{|c|c|c|c|}
\hline \multirow[t]{2}{*}{ Medication } & \multicolumn{2}{|c|}{ Recommendation } & \multirow[t]{2}{*}{ No recommendation } \\
\hline & Likely to remit & Not likely to remit & \\
\hline Escitalopram & $21 \%$ & $22 \%$ & $57 \%$ \\
\hline Sertraline & $24 \%$ & $39 \%$ & $37 \%$ \\
\hline Venlafaxine-XR & $21 \%$ & $27 \%$ & $52 \%$ \\
\hline
\end{tabular}

Abbreviation: $X R$, extended release. 
participants, both with and without cross-validation. Remission could be predicted with a cross-validated sensitivity of $72 \%$ and specificity of $67 \%$ for participants taking escitalopram with below-average emotion identification performance, with $69 \%$ sensitivity and $70 \%$ specificity in participants over 30 years of age taking sertraline, and with $67 \%$ sensitivity and $62 \%$ specificity in participants taking venlafaxine-XR with above average general cognitive performance.

Figure 2 shows the specific regression model and participant subgroup in each treatment arm. For participants taking escitalopram with below average emotion identification performance, the regression model used switching of attention completion time, verbal interference incongruent correct reaction time, and sustained attention reaction time as the predictors. For participants who were at least 30 years old taking sertraline, the regression model used switching of attention completion time, digit span forward trials correct, and number of maze overrun errors as the predictors. For participants with above average general cognition performance the regression model used baseline QIDS-SR ${ }_{16}$ severity as the predictor. No additional covariates were used in these regression models.

Figure 3 shows the receiver operator characteristics curves for each of these models using only the subset of participants in each medication group for whom a prediction of remission could be made. The test battery model for escitalopram and sertraline performed better than models that used age and sex or depression severity, as assessed by area under the curve. The test battery model for venlafaxine-XR performed better than a model that used age and sex, as assessed by area under the curve. While it was necessary to use baseline depression severity, defined by the QIDS-SR ${ }_{16}$, as a predictor of remission for participants with above average cognition performance taking venlafaxine-XR, baseline QIDS-SR ${ }_{16}$ did not predict remission in the full treatment arm for venlafaxine-XR (cross-validated sensitivity, specificity, PPV, and NPV: 61\%, $53 \%, 37 \%$, and $74 \%$ ) or in participants with below average general cognition performance (cross-validated sensitivity, specificity, PPV, and NPV: 38\%, 45\%, 21\%, and 66\%).

\section{Question 2}

Can the test battery identify a meaningful proportion of participants who will or will not achieve remission with a very high certainty level at least $80 \%$, with 8 weeks of escitalopram, sertraline or venlafaxine-XR.

Table 4 reports the proportion of participants for whom predictions of remission could be made with very high certainty (PPV $>80 \%$ ). The predicted probability of remission was obtained for participants in the specific subgroups using the specific regression equations reported in Figure 2. While PPV never surpassed $80 \%$ for either the sertraline or venlafaxine-XR models, a threshold was found in which PPV surpassed $80 \%$ for $22 / 157(14.0 \%)$ of the participants who received escitalopram.

Table 5 reports the proportion of participants for whom predictions of non-remission could be made with very high certainty (NPV $>80 \%$ ). The predicted probability of remission was obtained for participants in the specific subgroups using the regression equations reported in Figure 2. Overall, $24.2 \%(38 / 157), 53.7 \%(94 / 175)$, and $26.6 \%(36 / 135)$ of all participants in each treatment arm could be predicted to not remit with an NPV $>80 \%$ in escitalopram, sertraline, and venlafaxine-XR, respectively.

The test battery identified 38 of the 93 (40.9\%) participants who ultimately did not remit after 8 weeks of escitalopram, 94 of the $122(77.1 \%)$ participants who did not remit after 8 weeks of sertraline, and 36 of the $93(38.7 \%)$

Table 3 Sensitivity and specificity of the depression treatment test

\begin{tabular}{|c|c|c|c|c|c|c|}
\hline & Sensitivity & Specificity & PPV & NPV & Odds & NNT \\
\hline & $\begin{array}{l}\text { DT value } \\
\text { [chance rate] }\end{array}$ & $\begin{array}{l}\text { DT value } \\
\text { [chance rate] }\end{array}$ & $\begin{array}{l}\text { DT value [observed } \\
\text { remission rate] }\end{array}$ & $\begin{array}{l}\text { DT value [observed } \\
\text { non-remission rate] }\end{array}$ & & \\
\hline \multicolumn{7}{|l|}{ Full sample } \\
\hline Escitalopram & $75[50]$ & $70[50]$ & $64[4 I]$ & 80 [59] & 7.0 & 2.3 \\
\hline Sertraline & 7I [50] & $73[50]$ & 48 [25] & 88 [75] & 6.9 & 2.7 \\
\hline Venlafaxine-XR & $67[50]$ & $67[50]$ & 50 [33] & $81[67]$ & 4.1 & 3.3 \\
\hline \multicolumn{7}{|c|}{ Cross-validated } \\
\hline Escitalopram & $72[50]$ & $67[50]$ & $6 I[4 I]$ & 77 [59] & 4.9 & 2.7 \\
\hline Sertraline & $69[50]$ & $70[50]$ & 43 [25] & 87 [75] & 4.4 & 3.5 \\
\hline Venlafaxine-XR & $67[50]$ & $62[50]$ & 46 [33] & 81 [67] & 2.7 & 4.6 \\
\hline
\end{tabular}

Notes: All statistics reported here are calculated only for the intended population of the prediction model (better than average emotion identification in escitalopram, $>30$ years of age in sertraline, and better than average general cognition performance in venlafaxine-XR). Full-sample statistics report the accuracy statistics calculated from the same sample used to develop the regression model. Cross-validated statistics are the averaged accuracy statistics of 300 cross-validation iterations. Observed remission rates are based upon the specific cohort used for the depression test ( $\mathrm{n}=68, \mathrm{n}=\mathrm{I}$ I I, and $\mathrm{n}=64$ for escitalopram, sertraline and venlafaxine-XR, respectively). Abbreviations: DT, depression treatment test; NNT, number needed to treat; NPV, negative predictive values; PPV, positive predictive values; XR, extended release. 


\section{Escitalopram: $n=157$ included in analysis}

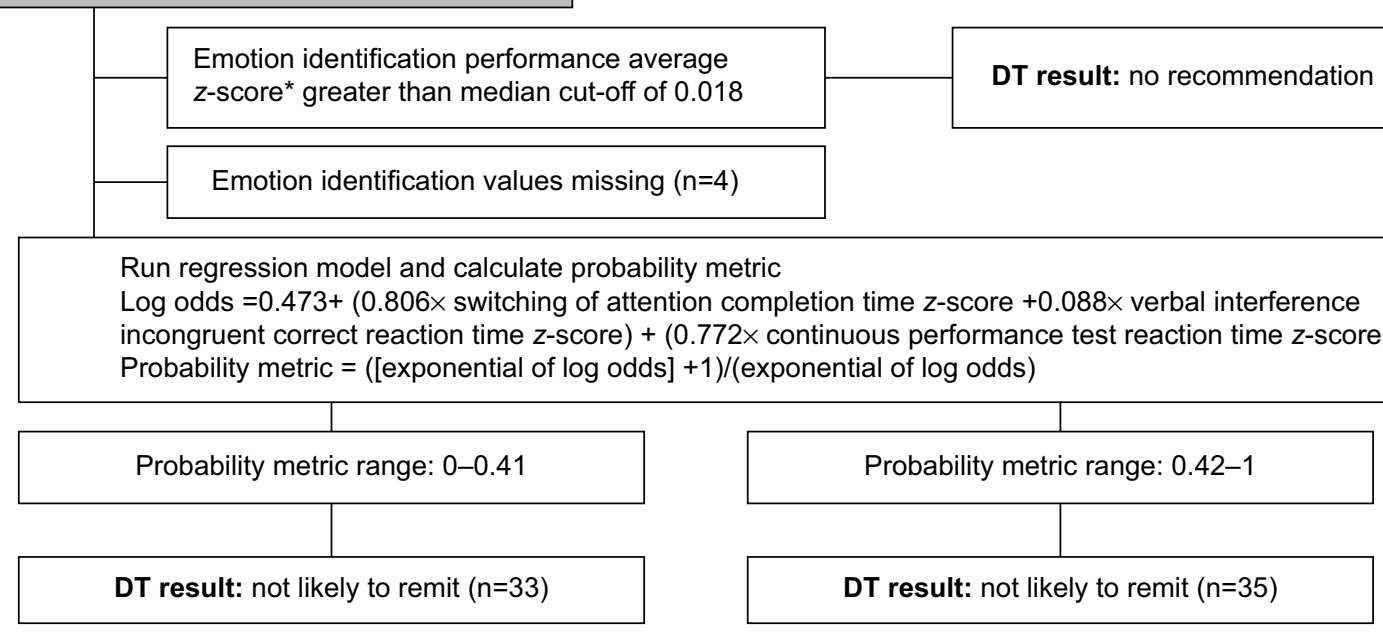

\section{Sertraline: $n=175$ included in analysis}

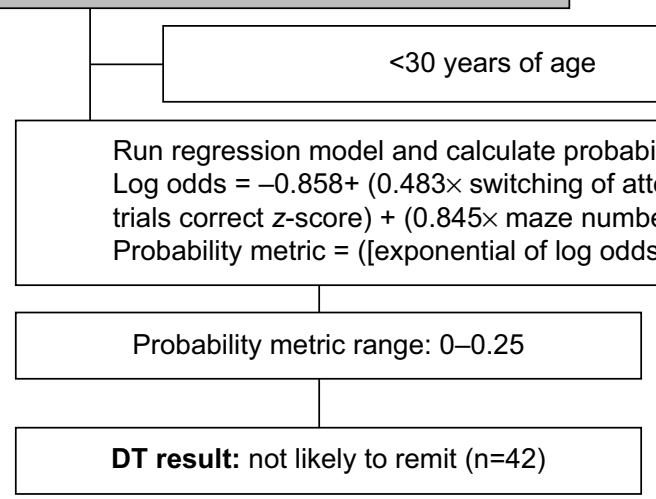

DT result: no recommendation $(n=64)$

Run regression model and calculate probability metric

列

trials correct $z$-score $)+(0.845 \times$ maze number of overruns $z$-score $)$

Probability metric $=([$ exponential of log odds $]+1) /($ exponential of log odds $)$

Probability metric range: $0-0.25$

DT result: not likely to remit $(n=42)$
Probability metric range: $0.26-1$

DT result: not likely to remit $(n=69)$

\section{Venlafaxine-XR: $n=135$ included in analysis}

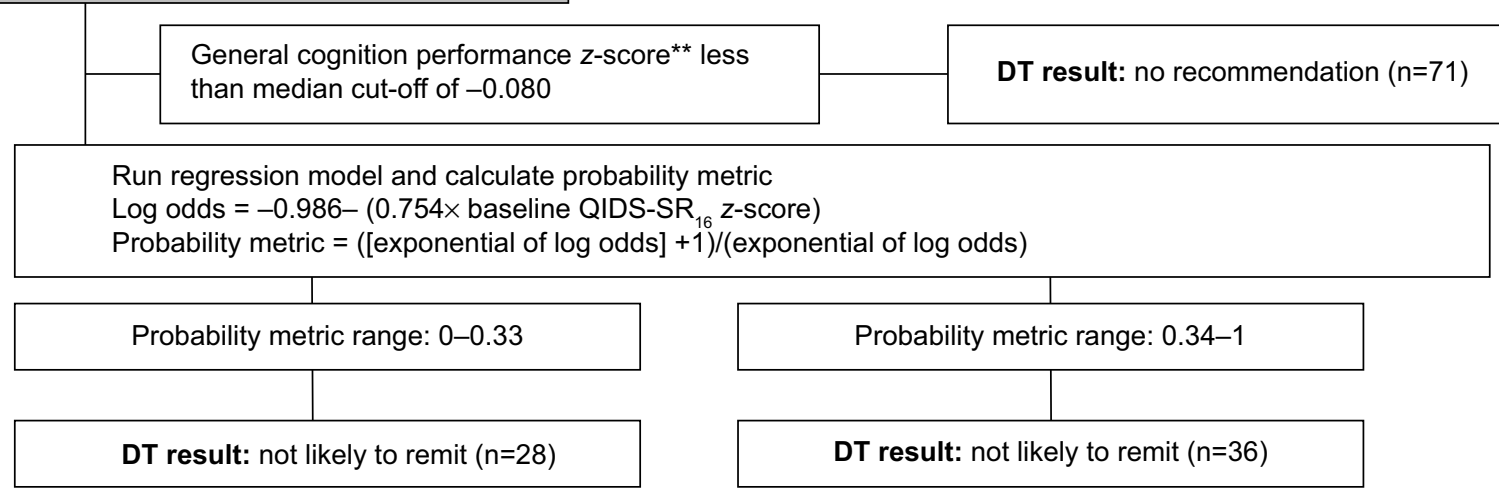

Figure 2 Final regression model to predict remission in each treatment arm and applicable subgroup.

Notes: Each treatment arm was stratified into two groups. Remission could be predicted 10 percentage points above chance. The specific subgroup and threshold is reported. The specific regression equation for each treatment arm is reported. Additionally, the number of patients in each treatment arm and each subgroup is also reported. DT result is the Depression Treatment Test predictive outcome. *Defined as average of $z$-scores for emotion identification number of correct responses and reaction time for correct responses. **Defined as average of z-scores for maze completion time, maze total number of errors and number of overrun errors, switching of attention completion time, verbal interference reaction time incongruent trials, continuous performance test reaction time, digit span total number of trials correct, and emotion identification number of correct response and reaction time for correct responses.

Abbreviations: DT, depression treatment test; QIDS-SR $_{16}$, 16-item Quick Inventory of Depressive Symptomatology Self-Report; XR, extended release. 
A

\section{Age and sex}

Logistic regression model

- Age and sex

\section{Escitalopram}

Logistic regression model $\mathrm{AUC}=0.78$ Age and sex model AUC $=0.65$

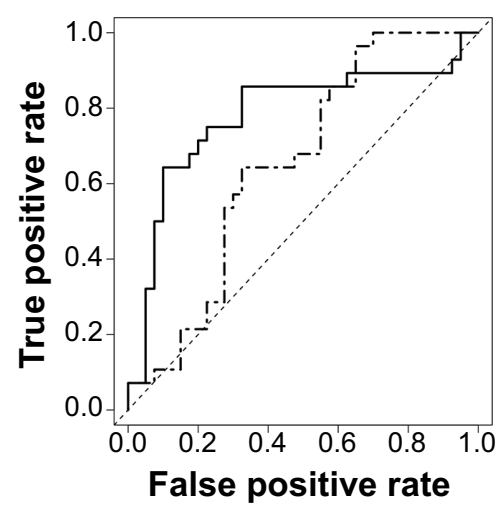

B

\section{Symptom severity}

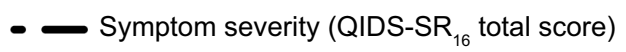

\section{Escitalopram}

Logistic regression model AUC $=0.78$ Symptom severity model AUC $=0.57$

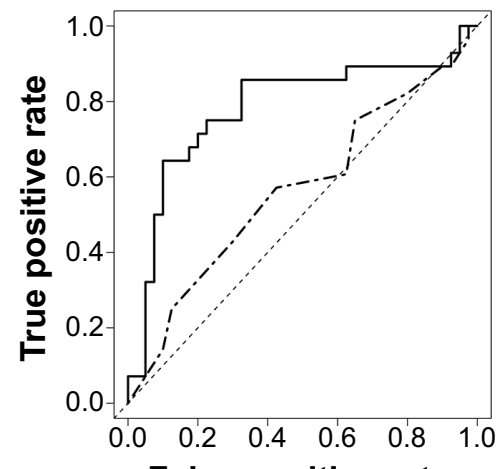

False positive rate

\section{Sertraline}

Logistic regression model AUC $=0.76$ Age and sex model AUC $=0.66$

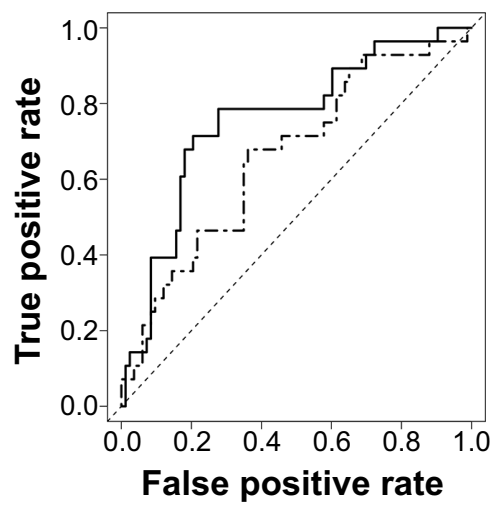

\section{Venlafaxine-XR}

Logistic regression model AUC $=0.67$

Age and sex model AUC $=0.59$

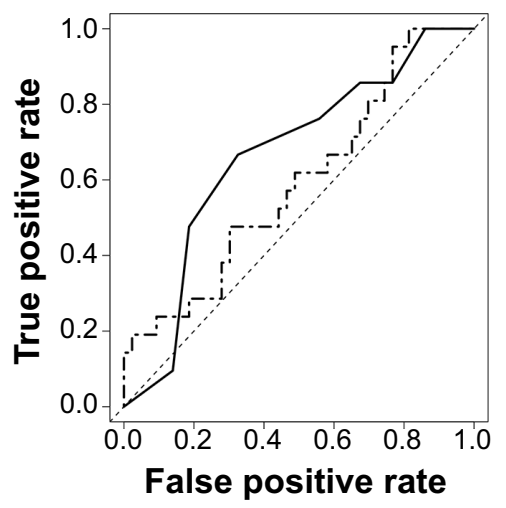

False positive rate 
Table 4 Proportion of patients identified with greater than $80 \%$ certainty to remit

\begin{tabular}{lllll}
\hline Medication & Total number & Total remitters & $\begin{array}{l}\text { Number of participants } \\
>\mathbf{8 0 \%} \text { PPV }\end{array}$ & $\begin{array}{l}\text { \% of total treatment } \\
\text { arm }\end{array}$ \\
\hline Escitalopram & 157 & 64 & 22 & $14.01 \%$ \\
Sertraline & 175 & 53 & - & - \\
Venlafaxine-XR & 135 & 42 & - & - \\
\hline
\end{tabular}

Notes: The proportion of participants identified by the regression models to be highly certain to remit (PPV $>80 \%$ ). The number of participants reported represents the total number of participants identified by the regression model to be highly certain to remit and hence will reflect both true and false positives. Percentages of remitters identified include false positives.

Abbreviations: PPV, positive predictive values; $X R$, extended release.

greater than chance. If one were to run all of the three tests developed for each treatment on all the patients, then a prediction of remission or non-remission could be made for $96 \%$ of the patients for at least one of the three medications.

The same test battery algorithm identified a meaningful proportion of participants who ultimately did not remit after 8 weeks of treatment with a very high level of certainty (at least $80 \%$ ). The test battery did not identify a meaningful proportion of participants in any treatment group who did remit with at least $80 \%$ certainty. On the other hand, the PPV were between $48 \%$ and $64 \%$ (cross-validated PPV were between $46 \%$ and $61 \%$ ), indicating a $17-23$ percentage point improvement from the remission rate. In other words, a participant who received a prediction of remission from any of the regression models would be at least 1.5-1.9 times as likely to remit compared to what would be expected by chance. Figure 4 illustrates the proportion of participants for whom a prediction could be made as well as the relative risks of predictions to remit and predictions to not remit. Moreover, different baseline test parameters were seen to predict remission in each of the three treatment arms. This finding is consistent with the idea that the patients who remitted on each medication may not be identical, though there may be some overlap among the three groups of remitting patients.

Present results are consistent with the general notion that cognitive measures can differentiate depressed patients who respond or remit acutely from those who do not. Dunkin et $\mathrm{al}^{33}$ found that non-responders to fluoxetine performed significantly worse than responders on the Wisconsin Card Sorting Test (executive function) at baseline. Alexopoulos et $\mathrm{al}^{34}$ found among geriatric patients, non-responders to citalopram had poorer scores in the initiation/perseveration subscale of the Mattis Dementia Rating Scale and lower performance in the Stroop test than responders. Taylor et $\mathrm{al}^{8}$ reported that non-responders to fluoxetine displayed psychomotor slowing, measured with the Stroop and fluency tests. Gorlyn et a ${ }^{35}$ found that responders treated with various selective serotonin reuptake inhibitors (SSRIs) outperformed non-responders across all cognitive domains, with the largest differences observed in executive function, language and working memory. Kampf-Sherf et $\mathrm{al}^{36}$ found that responders to various SSRIs performed better on "simple" tasks and worse on "complex" tasks on a small cognitive battery that measured memory and executive functions. Gudayol-Ferré et a ${ }^{17}$ found that response to fluoxetine was predicted by good performance in variables of attention and low performance in planning. Conversely, Herrera-Guzmán et $\mathrm{al}^{37}$ found that bupropion responders displayed low pre-treatment measures of visual memory and low levels of mental processing speed. Taken together, these studies reveal an association between baseline cognitive function and acute treatment outcomes. However, these studies assert group differences, which do not make individual patient predictions, and are, therefore, unable to guide individual treatment selection.

This report, however, goes beyond most of the above literature in that it aims to address the challenge of making

Table 5 Proportion of patients identified with greater than $80 \%$ certainty to not remit

\begin{tabular}{llllc}
\hline Medication & Total number & Total non-remitters & $\begin{array}{l}\text { Number of participants } \\
>\mathbf{8 0 \%} \text { NPV }\end{array}$ & $\begin{array}{c}\text { \% of total } \\
\text { treatment arm }\end{array}$ \\
\hline Escitalopram & 157 & 93 & 38 & $24.20 \%$ \\
Sertraline & 175 & 122 & 94 & $53.71 \%$ \\
Venlafaxine-XR & 135 & 93 & 36 & $77.05 \%$ \\
\hline
\end{tabular}

Notes: The proportion of participants identified by the regression models to be highly certain to not remit (NPV $>80 \%)$. The number of participants reported represents the total number of participants identified by the regression model to be highly certain to remit and hence will reflect both true and false negatives. Percentages of nonremitters identified include false positives.

Abbreviations: NPV, negative predictive values; XR, extended release. 


\section{Percent of sample Relative risk}

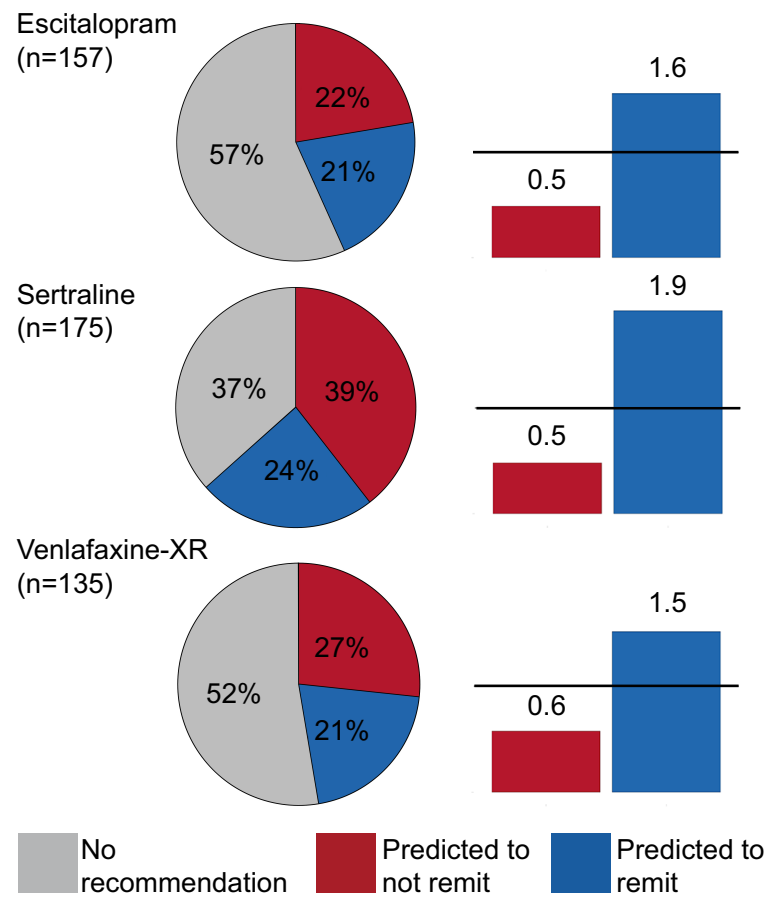

Figure 4 Percent of the sample receiving a recommendation to remit or to not remit, and relative risks for each type of prediction.

Notes: Relative risks were calculated using the remission rate for the specific patients for whom a prediction was made. A horizontal bar is drawn at a relative risk of I to allow for chance comparisons. Patients predicted to not remit, with a relative risk of $0.5-0.6$, have one half the remission rate for the subgroup used to develop the specific regression model. Patients predicted to remit, with a relative risk of I.5-1.9, have one and a half to two times the remission rate for the subgroup used to develop the specific regression model.

Abbreviation: $X R$, extended release.

individual patient predictions. In this context, the test battery was able to identify individual participants for whom recommendations could be made, outperforming chance for almost half of the participants. These findings are consistent with Etkin et al ${ }^{38}$ who used a novel pattern classification analysis to show that predictions of remission can be attained for a single subgroup of one quarter of patients who show widespread cognitive deficits. This current report, focused on whether predictions of remission using measures of cognition can be obtained for a larger proportion of the patient population by using simpler statistical techniques that are more clinically actionable by pragmatically limiting the number of informative parameters needed to make predictions. With this method, the test battery was able to identify $32 \%-62 \%$ of individual participants who will not remit after 8 weeks of treatment with an actionable level of certainty $(80 \%)$.

These findings are also consistent with a conceptual point raised by Kapur et $\mathrm{al}^{39}$ and Prata et al. ${ }^{40}$ They note that "diagnostic biomarkers themselves could be used to identify meaningful clinical sub-phenotypes" (Prata et al). ${ }^{40}$
In fact, the test battery itself was initially formulated based on parameters that were presumed to differentiate depressed individuals from controls. Thus potentially "diagnostic" test elements may also identify treatment outcomes in subgroups of individuals. In addition, this report shows that different elements in the test battery identify individuals who remit or do not remit, suggesting that different patients are remitting with different treatments, despite the likely contribution of nonspecific treatment effects that are likely present in each group of participants who remitted.

This report has several strengths and limitations. The sample sizes are substantial. Data come from multiple sites suggesting generalizability. Analyses focused on the per protocol completer sample rather than on the full intent-to-treat sample to be certain that each individual participant whose outcome we wished to predict completed the treatment. This approach limits the generalizability of the findings and it may exaggerate or underestimate the value of the cognitive and emotional test battery as those who complete the full 8 weeks tend to be those who are benefitting. Additionally, the study exclusion criteria limit the generalizability. Further study will be required to determine how the test battery would actually perform in practice. However, the cross-validated analyses provide estimates of model performance on data not used to develop the model thereby providing an assessment of the generalizability of these models to new patients. Second, the doses of the medications used in the study were modest (mean final dosages: $12.3 \mathrm{mg}$ for escitalopram, $63.6 \mathrm{mg}$ for sertraline, and $84.9 \mathrm{mg}$ for venlafaxine-XR), which may be representative of typical but not optimal patient care in diverse treatment settings. Higher medication doses might have resulted in more remitted participants which could affect the performance of the test battery. There is a clear need to replicate and extend these findings in new samples to evaluate the reliability, generalizability, and validity of these results, as noted in Williams et al. ${ }^{26}$

In conclusion, this report suggests that, if replicated, a cognitive and emotional test battery can be clinically useful in identifying individual depressed patients who will remit or not remit beyond chance expectations. For all three medications, a meaningful number of individuals who did not remit was identified at baseline, with sufficient certainty that the clinician can decide to consider alternative treatments.

\section{Acknowledgments}

The iSPOT-D study is sponsored by Brain Resource Company Operations Pty Ltd. We received financial and material support from Brain Resource Ltd., Sydney, NSW, Australia. 
We received material support from Brain Resource Inc., San Francisco, CA, USA. We gratefully acknowledge the iSPOT-D Investigators Group, and the contributions of principal investigators at each site, the monitoring support of Claire Day and the central iSPOT-D management team and PhaseForward, the data processing and quality control support of the central iSPOT-D management team, the secretarial support of Liane C Kivela, the editorial support of Jon Kilner, MS, MA (Pittsburgh, PA), and the support of Leanne William $\mathrm{PhD}$ in the design and execution of the iSPOT-D study.

\section{Trial registry}

Registry Name: ClinicalTrials.gov. Registration Number: NCT00693849. URL: http://www.clinicaltrials.gov/ct2/ show/NCT00693849?term=ispot-D\&rank=1.

\section{Disclosure}

Drs Gordon and Palmer, Mr Braund and Mr Rekshan are employees of Brain Resource Ltd. Dr Palmer, Mr Braund, and Mr Rekshan hold stock options in Brain Resource Ltd. In the last 3 years, Dr Rush has received consulting fees from Brain Resource Ltd, H. Eli Lilly, Lundbeck A/S, Medavante, Inc; National Institute of Drug Abuse, Santium Inc., Takeda USA; speaking fees from the University of California at San Diego, Hershey Penn State Medical Center, the American Society for Clinical Psychopharmacology, and New York State Psychiatric Inst; royalties from Guilford Publications and from the University of Texas Southwestern Medical Center; a travel grant from CINP and research support from Duke-National University of Singapore. The authors have no other conflicts of interest to declare.

\section{References}

1. Kessler RC, Berglund P, Demler O, et al. The epidemiology of major depressive disorder: results from the National Comorbidity Survey Replication (NCS-R). JAMA. 2003;289(23):3095-3105.

2. World Health Organization. The global burden of disease: 2004 update. Geneva: WHO; 2008.

3. World Health Organization [homepage on the Internet]. Mental Health Atlas 2011. Geneva, Switzerland: WHO; c2011. Available from: http:// www.who.int/mental_health/publications/mental_health_atlas_2011/en/ index.html. Accessed 8 September 2014.

4. Rush AJ, Trivedi MH, Wisniewski SR, et al. Acute and longer-term outcomes in depressed outpatients requiring one or several treatment steps: a STAR*D report. Am J Psychiatry. 2006;163(11):1905-1917.

5. Rush AJ, Kraemer HC, Sackeim HA, et al. Report by the ACNP Task Force on response and remission in major depressive disorder. Neuropsychopharmacology. 2006;31(9):1842-1853.

6. Trivedi MH, Rush AJ, Wisniewski SR, et al. Evaluation of outcomes with citalopram for depression using measurement-based care in STAR*D: implications for clinical practice. Am J Psychiatry. 2006;163(1):28-40.

7. Thase ME, Rush AJ. Treatment-resistant depression. In: Bloom FE, Kupfer DJ, editors. Psychopharmacology: fourth generation of progress. New York: Raven Press; 1995:1081-1097.
8. Taylor BP, Bruder GE, Stewart JW, et al. Psychomotor slowing as a predictor of fluoxetine nonresponse in depressed outpatients. Am J Psychiatry. 2006;163(1):73-78.

9. O'Leary D, Costello F, Gormley N, Webb M. Remission onset and relapse in depression. An 18-month prospective study of course for 100 first admission patients. $J$ Affect Disord. 2000;57(1-3):159-171.

10. Rush AJ. STAR*D: what have we learned? Am J Psychiatry. 2007;164(2):201-204

11. Bagby RM, Ryder AG, Cristi C. Psychosocial and clinical predictors of response to pharmacotherapy for depression. J Psychiatry Neurosci. 2002;27(4):250-257.

12. Gaynes BN, Warden D, Trivedi MH, Wisniewski SR, Fava M, Rush AJ. What did STAR*D teach us? Results from a large-scale, practical, clinical trial for patients with depression. Psychiatr Serv. 2009;60(11):1439-1445.

13. Kuk AY, Li J, Rush AJ. Recursive subsetting to identify patients in the STAR*D: a method to enhance the accuracy of early prediction of treatment outcome and to inform personalized care. J Clin Psychiatry. 2010;71(11):1502-1508.

14. Li J, Kuk AY, Rush AJ. A practical approach to the early identification of antidepressant medication non-responders. Psychol Med. 2012;42(2):309-316.

15. Lutz W, Lowry J, Kopta SM, Einstein DA, Howard KI. Prediction of dose-response relations based on patient characteristics. J Clin Psychol. 2001;57(7):889-900.

16. Simon GE, Khandker RK, Ichikawa L, Operskalski BH. Recovery from depression predicts lower health services costs. J Clin Psychiatry. 2006;67(8):1226-1231.

17. Gudayol-Ferre E, Herrera-Guzman I, Camarena B, et al. The role of clinical variables, neuropsychological performance and SLC6A4 and COMT gene polymorphisms on the prediction of early response to fluoxetine in major depressive disorder. $J$ Affect Disord. 2010;127(1-3): 343-351.

18. Korgaonkar MS, Williams LM, Song YJ, Usherwood T, Grieve SM. Diffusion tensor imaging predictors of treatment outcomes in major depressive disorder. Br J Psychiatry. 2014;205(4):321-328.

19. No authors listed. Practice guideline for the treatment of patients with major depressive disorder (revision). American Psychiatric Association. Am J Psychiatry. 2000;157(4 Suppl):1-45.

20. NCCMH. Depression: The Treatment and Management of Depression in Adults (Updated Edition). Leicester and London: The British Psychological Society and the Royal College of Psychiatrists; 2010.

21. Rush AJ, Wisniewski SR, Warden D, et al. Selecting among second-step antidepressant medication monotherapies: predictive value of clinical, demographic, or first-step treatment features. Arch Gen Psychiatry. 2008;65(8):870-880.

22. Gordon E, Cooper N, Rennie C, Hermens D, Williams LM. Integrative neuroscience: the role of a standardized database. Clin EEG Neurosci. 2005;36(2):64-75.

23. Paul RH, Lawrence J, Williams LM, Richard CC, Cooper N, Gordon E. Preliminary validity of "integneuro": a new computerized battery of neurocognitive tests. Int J Neurosci. 2005;115(11):1549-1567.

24. Paul RH, Gunstad J, Cooper N, et al. Cross-cultural assessment of neuropsychological performance and electrical brain function measures: additional validation of an international brain database. Int $J$ Neurosci. 2007;117(4):549-568

25. Williams LM, Mathersul D, Palmer DM, Gur RC, Gur RE, Gordon E. Explicit identification and implicit recognition of facial emotions: I. Age effects in males and females across 10 decades. J Clin Exp Neurpsychol. 2009;31(3):257-277.

26. Williams LM, Rush AJ, Koslow SH, et al. International Study to Predict Optimized Treatment for Depression (iSPOT-D), a randomized clinical trial: rationale and protocol. Trials. 2011;12:4.

27. Rush AJ, Nierenberg A. Mood disorders: Treatment of depression. In: Sadock BJ, Sadock VA, Ruiz P, editors. Kaplan \& Sadock's Comprehensive Textbook of Psychiatry, Ninth Edition. Philadelphia, Pennsylvania: Lippincott Williams \& Wilkins; 2009. 
28. Maroco J, Silva D, Rodrigues A, Guerreiro M, Santana I, Mendonca A. Data mining methods in the prediction of Dementia: A real-data comparison of the accuracy, sensitivity and specificity of linear discriminate analysis, logistic regression, neural networks, support vector machines, calcification tress and random forests. BMC Research Notes. 2011;4:299.

29. R Core Team. R: A language and environment for statistical computing. Vienna, Austria: R Foundation for Statistical Computer; 2013. Available from: http://www.R-project.org/. Accessed September 25, 2013.

30. Kuhn M. caret: Classification and regression training. $\mathrm{R}$ package version 5.15-7. 2013. Available from: http://CRAN.R-project.org/ package $=$ caret. Accessed September 14, 2013.

31. Carstensen B, Plummer M, Laara E, Hills M. Epi: A package for statistical analysis in epidemiology. R package version 1.1.49. 2013. Available from: http://CRAN.R-project.org/package=Epi. Accessed September 14, 2013.

32. Wickham H. ggplot2: elegant graphics for data analysis. New York: Springer; 2009.

33. Dunkin JJ, Leuchter AF, Cook IA, Kasl-Godley JE, Abrams M, Rosenberg-Thompson S. Executive dysfunction predicts nonresponse to fluoxetine in major depression. J Affect Disord. 2000;60(1):13-23.

34. Alexopoulos GS, Kiosses DN, Heo M, Murphy CF, Shanmugham B, Gunning-Dixon F. Executive dysfunction and the course of geriatric depression. Biol Psychiatry. 2005;58(3):204-210.
35. Gorlyn M, Keilp JG, Grunebaum MF, et al. Neuropsychological characteristics as predictors of SSRI treatment response in depressed subjects. J Neural Transm. 2008;115(8):1213-1219.

36. Kampf-Sherf O, Zlotogorski Z, Gilboa A, et al. Neuropsychological functioning in major depression and responsiveness to selective serotonin reuptake inhibitors antidepressants. J Affect Disord. 2004;82(3):453-459.

37. Herrera-Guzman I, Gudayol-Ferre E, Lira-Mandujano J, et al. Cognitive predictors of treatment response to bupropion and cognitive effects of bupropion in patients with major depressive disorder. Psychiatry Res. 2008;160(1):72-82.

38. Etkin A, Patenaude B, Song Y, et al. A Cognitive-Emotional Biomarker for Predicting Remission with Antidepressant Medications: A Report from the iSPOT-D Trial. Neuropharmacology. In press.

39. Kapur S, Phillips AC, Insel TR. Why has it taken so long for biological psychiatry to develop clinical tests and what to do about it? Mol Psychiatry. 2012;17(12):1174-1179.

40. Prata D, Mechelli A, Kapur S. Clinically meaningful biomarkers for psychosis: A systematic and quantitative review. Neurosci Biobehav Rev. 2014;45:134-141. 


\section{Supplementary materials}

Table SI Inclusion/exclusion criteria

Inclusion criteria

- Age 18-65

- Fluent and literate in English or Dutch

- Provide written informed consent

- Total $\mathrm{HRSD}_{17} \geq 16$

- Meets DSM-IV criteria for single or recurrent nonpsychotic MDD established by MINI Plus

Exclusion criteria

- Suicidal ideation and/or tendencies, defined by a score $\geq 8$ on section $C$ of the MINI Plus

- History of bipolar disorder (I, II, not otherwise specified) (lifetime)

- History of schizophrenia, schizoaffective disorder, or psychosis not otherwise specified (lifetime)

- Current primary diagnosis of anorexia or bulimia, obsessive-compulsive disorder, or primary post-traumatic stress disorder

- Known contraindication for escitalopram, sertraline and/or venlafaxine-XR, or previous treatment failure at the highest recommended dose

- Taking any medication that is contraindicated with escitalopram, sertraline, or venlafaxine-XR

- Taking escitalopram, sertraline, or venlafaxine-XR in the current episode of MDD

- Use of any non-protocol antidepressant drug or CNS drug (antipsychotic, anticonvulsant anxiolytic, clonidine) that cannot be washed out prior to participation

- Has general medical condition that contraindicates protocol antidepressant treatments or interferes with protocol measurements (such as epileptic condition for EEG recording)

- Substance dependence (including alcohol intake equaling 29 standard alcoholic drinks per week for males; > 15 for females) in the past 6 months

- History of brain injury or blow to the head that resulted in loss of consciousness for greater than 5 minutes

- Severe impediment to vision, hearing and/or hand movement that is likely to interfere with completion of assessments, or with comprehension of instructions or study requirements

- Participation in an investigational study within 4 months prior to baseline that could affect symptoms of MDD

- Is pregnant or breast-feeding

Abbreviations: $\mathrm{HRSD}_{17}$, 17-item Hamilton Rating Scale for Depression; MDD, major depressive disorder; XR, extended release; EEG, electroencephalogram; DSM, Diagnostic and Statistical Manual of Mental Disorders; MINI, Mini-International Neuropsychiatric Interview; CNS, central nervous system. 
Table S2 Comparison of included and excluded samples (categorical measures)

\begin{tabular}{|c|c|c|c|c|c|c|c|c|}
\hline & \multicolumn{3}{|c|}{ Excluded from sample $(\mathrm{N}=54 \mathrm{I})$} & \multicolumn{3}{|c|}{ Included in sample $(\mathrm{N}=467)$} & \multirow[t]{2}{*}{$\chi^{2}$} & \multirow[t]{2}{*}{$P$-value } \\
\hline & Count & $\%$ & Total $\mathbf{N}$ & Count & $\%$ & Total $\mathbf{N}$ & & \\
\hline Sex (female) & 240 & $44.36 \%$ & 541 & 197 & $42.18 \%$ & 467 & 3.498 & 0.174 \\
\hline Family history of MDD & 114 & $21.07 \%$ & 541 & 119 & $25.48 \%$ & 467 & 0.313 & 0.855 \\
\hline MDD diagnosis prior to 18 & 239 & $45.01 \%$ & 531 & 239 & $51.96 \%$ & 460 & 1.992 & 0.369 \\
\hline Race & & & & & & & 5.146 & 0.161 \\
\hline Black & 93 & $17.19 \%$ & 541 & 74 & $15.85 \%$ & 467 & & \\
\hline Other & 125 & $23.11 \%$ & 541 & 87 & $18.63 \%$ & 467 & & \\
\hline Unknown & 1 & $0.18 \%$ & 541 & 3 & $0.64 \%$ & 467 & & \\
\hline White & 322 & $59.52 \%$ & 541 & 303 & $64.88 \%$ & 467 & & \\
\hline Employment & & & & & & & 6.848 & 0.232 \\
\hline Employed & 267 & $49.35 \%$ & 541 & 239 & $51.18 \%$ & 467 & & \\
\hline Other & 37 & $6.84 \%$ & $54 I$ & 36 & $7.71 \%$ & 467 & & \\
\hline Retired & 28 & $5.18 \%$ & 541 & 15 & $3.21 \%$ & 467 & & \\
\hline Student & 100 & $18.48 \%$ & 541 & 89 & $19.06 \%$ & 467 & & \\
\hline Unemployed & 30 & $5.55 \%$ & 541 & 36 & $7.71 \%$ & 467 & & \\
\hline Unknown & 79 & $14.60 \%$ & $54 I$ & 52 & $11.13 \%$ & 467 & & \\
\hline Marital status & & & & & & & 6.391 & 0.172 \\
\hline Divorced/separated & 68 & $12.57 \%$ & 541 & 74 & $15.85 \%$ & 467 & & \\
\hline Married/cohabiting & 98 & $18.11 \%$ & 541 & 95 & $20.34 \%$ & 467 & & \\
\hline Single & 341 & $63.03 \%$ & 541 & 273 & $58.46 \%$ & 467 & & \\
\hline Unknown & 27 & $4.99 \%$ & 541 & 15 & $3.21 \%$ & 467 & & \\
\hline Widowed & 7 & $1.29 \%$ & 541 & 10 & $2.14 \%$ & 467 & & \\
\hline Recruitment source & & & & & & & 1.261 & 0.738 \\
\hline Ad & 424 & $78.37 \%$ & 541 & 371 & $79.44 \%$ & 467 & & \\
\hline GP & 12 & $2.22 \%$ & 541 & 14 & $3.00 \%$ & 467 & & \\
\hline Other & 48 & $8.87 \%$ & 541 & 40 & $8.57 \%$ & 467 & & \\
\hline Psych & 57 & $10.54 \%$ & 541 & 42 & $8.99 \%$ & 467 & & \\
\hline Number of episodes & & & & & & & 6.298 & 0.278 \\
\hline 0 & 2 & $0.38 \%$ & 528 & 0 & $0.00 \%$ & 457 & & \\
\hline 1 & 57 & $10.80 \%$ & 528 & 46 & $10.07 \%$ & 457 & & \\
\hline 2 & 53 & $10.04 \%$ & 528 & 32 & $7.00 \%$ & 457 & & \\
\hline 3 & 60 & $11.36 \%$ & 528 & 45 & $9.85 \%$ & 457 & & \\
\hline 4 & 64 & $12.12 \%$ & 528 & 56 & $12.25 \%$ & 457 & & \\
\hline 5 or more & 292 & $55.30 \%$ & 528 & 278 & $60.83 \%$ & 457 & & \\
\hline Dysthymia & 101 & $18.67 \%$ & 541 & 118 & $25.27 \%$ & 467 & 2.660 & 0.264 \\
\hline Panic disorder & 43 & $7.95 \%$ & $54 I$ & 42 & $8.99 \%$ & 467 & 0.488 & 0.783 \\
\hline Agoraphobia & 37 & $6.85 \%$ & 540 & 37 & $7.92 \%$ & 467 & 1.237 & 0.539 \\
\hline Social phobia & 37 & $6.84 \%$ & 541 & 56 & $11.99 \%$ & 467 & 4.549 & 0.103 \\
\hline Specific phobia & 24 & $4.44 \%$ & 541 & 31 & $6.65 \%$ & 466 & 0.607 & 0.738 \\
\hline Generalized anxiety disorder & 33 & $6.10 \%$ & 541 & 36 & $7.71 \%$ & 467 & 3.360 & 0.186 \\
\hline Previous suicide attempt & 64 & $11.83 \%$ & 541 & 53 & $11.35 \%$ & 467 & 0.406 & 0.816 \\
\hline \multicolumn{4}{|c|}{ Number of comorbid medication conditions } & & & & $|5.37|$ & 0.009 \\
\hline 0 & 333 & $61.55 \%$ & 541 & 236 & $50.54 \%$ & 467 & & \\
\hline 1 & 111 & $20.52 \%$ & 541 & 122 & $26.12 \%$ & 467 & & \\
\hline 2 & 51 & $9.43 \%$ & 541 & 45 & $9.64 \%$ & 467 & & \\
\hline 3 & 22 & $4.07 \%$ & 541 & 27 & $5.78 \%$ & 467 & & \\
\hline 4 & 12 & $2.22 \%$ & 541 & 19 & $4.07 \%$ & 467 & & \\
\hline 5 or more & 12 & $2.22 \%$ & $54 I$ & 18 & $3.85 \%$ & 467 & & \\
\hline MDD recurrence & & & & & & & 0.393 & 0.822 \\
\hline Not recurrent & 59 & $10.91 \%$ & 541 & 46 & $9.85 \%$ & 467 & & \\
\hline Recurrent & 469 & $86.69 \%$ & 541 & 411 & $88.01 \%$ & 467 & & \\
\hline Unknown & 13 & $2.40 \%$ & 541 & 10 & $2.14 \%$ & 467 & & \\
\hline
\end{tabular}

Abbreviations: MDD, major depressive disorder; GP, general practitioner; Psych, psychiatrist/psychologist. 
Table S3 Comparison of included and excluded samples (continuous measures)

\begin{tabular}{|c|c|c|c|c|c|c|c|c|}
\hline & \multicolumn{3}{|c|}{ Excluded from sample $(\mathrm{N}=54 \mathrm{I})$} & \multicolumn{3}{|c|}{ Included in sample ( $\mathrm{N}=467)$} & \multirow[t]{2}{*}{$t$} & \multirow[t]{2}{*}{$P$-value } \\
\hline & Mean & SD & Total N & Mean & SD & Total $\mathbf{N}$ & & \\
\hline Age (years) & 37.30 & 12.56 & 541 & 38.47 & 12.57 & 467 & -1.468 & 0.142 \\
\hline Education (years) & 14.56 & 2.73 & 541 & 14.52 & 2.88 & 467 & 0.189 & 0.850 \\
\hline Age at first episode (years) & 23.21 & 12.20 & 531 & 22.54 & 11.84 & 460 & 0.873 & 0.383 \\
\hline Duration of MDD (years) & 14.02 & 11.64 & 531 & 15.83 & 12.72 & 460 & -2.323 & 0.020 \\
\hline $\mathrm{HRSD}_{17}$ score & 21.58 & 4.11 & 541 & 22.24 & 4.12 & 467 & -2.521 & 0.012 \\
\hline QIDS-SR $_{16}$ score & 13.15 & 4.22 & 496 & 15.86 & 2.72 & 467 & -11.920 & 0.000 \\
\hline DASS (depression) & 19.73 & 9.45 & 469 & 24.75 & 8.97 & 451 & -8.255 & 0.000 \\
\hline DASS (anxiety) & 8.08 & 6.71 & 469 & 9.49 & 6.63 & 451 & -3.199 & 0.001 \\
\hline DASS (stress) & 17.00 & 8.15 & 469 & 19.47 & 8.45 & 451 & -4.498 & 0.000 \\
\hline SOFAS & 56.34 & 9.36 & 540 & 55.40 & 8.79 & 466 & 1.633 & 0.103 \\
\hline SWLS & 12.10 & 5.51 & 506 & 11.01 & 5.17 & 461 & 3.184 & 0.002 \\
\hline WHOQoL-physical & 54.44 & 14.04 & 485 & 49.02 & 14.35 & 453 & 5.850 & 0.000 \\
\hline WHOQoL-psychological & 37.19 & 14.29 & 484 & 31.93 & 12.79 & 453 & 5.948 & 0.000 \\
\hline WHOQoL-social & 40.96 & 20.21 & 485 & 36.09 & 19.22 & 454 & 3.784 & 0.000 \\
\hline WHOQoL-environmental & 52.17 & 15.64 & 483 & 51.18 & 15.93 & 451 & 0.958 & 0.338 \\
\hline WHOQoL-overall & 2.84 & 0.92 & 486 & 2.65 & 0.93 & 457 & 3.299 & 0.001 \\
\hline ERQ (reappraisal) & 4.39 & 1.24 & 506 & 4.26 & 1.22 & 465 & 1.662 & 0.097 \\
\hline ERQ (suppression) & 4.09 & 1.34 & 506 & 4.22 & 1.35 & 465 & -1.557 & 0.120 \\
\hline
\end{tabular}

Abbreviations: DASS, depression anxiety stress scale; ERQ, emotion regulation questionnaire; HRSD, 17-item Hamilton Rating Scale for Depression; MDD, major depressive disorder; QIDS-SR ${ }_{16}$, 16-item Quick Inventory of Depressive Symptomatology Self-Report; SOFAS, social and occupational functioning assessment scale; SWLS, satisfaction with life scale; WHOQoL, World Health Organization quality of life; SD, standard deviation.

\section{Publish your work in this journal}

Neuropsychiatric Disease and Treatment is an international, peerreviewed journal of clinical therapeutics and pharmacology focusing on concise rapid reporting of clinical or pre-clinical studies on a range of neuropsychiatric and neurological disorders. This journal is indexed on PubMed Central, the 'PsycINFO' database and CAS, and is the official journal of The International Neuropsychiatric Association (INA). The manuscript management system is completely online and includes a very quick and fair peer-review system, which is all easy to use. Visit http://www.dovepress.com/testimonials.php to read real quotes from published authors. 